PROCEEDINGS OF THE

AMERICAN MATHEMATICAL SOCIETY

Volume 129, Number 9 , Pages 2789-2797

S 0002-9939(01)06068-3

Article electronically published on February 9, 2001

\title{
DETERMINACY OF SMOOTH GERMS WITH REAL ISOLATED LINE SINGULARITIES
}

\author{
BOHAO SUN AND LESLIE C. WILSON
}

(Communicated by Jozef Dodziuk)

\begin{abstract}
The germ of a smooth real-valued function on Euclidean space is called a real isolated line singularity if its singular set is a nonsingular curve, its Jacobian ideal is Łojasiewicz at the singular set, and its Hessian determinant restricted to the singular set is Łojasiewicz at 0 . Consider the set of all germs whose singular set contains a fixed nonsingular curve $L$. We prove that such a germ $f$ is infinitely determined among all such germs with respect to composition by diffeomorphisms preserving $L$ if, and only if, the Jacobian ideal of $f$ contains all germs which vanish on $L$ and are infinitely flat at 0 if, and only if, $f$ is a real isolated line singularity.
\end{abstract}

\section{§1. INTRODUCTION}

If $f$ is a complex holomorphic germ in $n$ variables with an isolated singularity at 0 , then $V(f):=f^{-1}(0)$ is a hypersurface germ with an isolated singularity at 0 . These of course have been widely studied. One of the properties of such an $f$ is that it is finitely determined: there is a $k$ such that $j^{k} g(0)=j^{k} f(0)$ implies that there is a germ of a biholomorphic $h$ such that $g \circ h=f$ (i.e., $g$ is "right-equivalent" to $f$ ). More recently there has been much interest in studying varieties with non-isolated singularities. The simplest such varieties are the zero sets of an $f$ whose singular set $\Sigma(f)$ is a nonsingular curve with transversal cross-section away from 0 a Morse singularity; these are called "isolated line singularities". Siersma, in [Si], proved the finite determinacy of these inside the space of functions whose singular sets contain $\Sigma(f)$. In this paper we prove the analogue of this latter result for real analytic or smooth functions.

Let $\mathbf{E}_{k}$ be the ring of all germs at 0 of smooth functions on $\mathbf{R}^{k}, \mathbf{m}_{k}$ be the maximal ideal of $\mathbf{E}_{k}$ and $J_{k}^{l}=\mathbf{E}_{k} / \mathbf{m}_{k}^{l+1}$ be the jet space. Denote by $\mathcal{R}$ the group of all smooth local diffeomorphisms $\phi$ at 0 on $\mathbf{R}^{k}$ which fix the origin, i.e., $\phi(0)=0$. $\mathcal{R}$ acts on $\mathbf{E}_{k}$ by composition; $f, g \in \mathbf{E}_{k}$ are $\mathcal{R}$-equivalent if they are in the same $\mathcal{R}$ orbit. One says that $f$ is finitely (respectively, infinitely) $\mathcal{R}$-determined if there is an $l \in \mathbf{N}$ (respectively, $l=\infty$ ) such that $j^{l} g(0)=j^{l} f(0)$ implies that $g$ and $f$ are $\mathcal{R}$-equivalent. If $f$ is a function of $x_{1}, \ldots, x_{k}$, let $J(f)$ denote the Jacobian ideal $\left(\frac{\partial f}{\partial x_{1}}, \ldots, \frac{\partial f}{\partial x_{k}}\right) \mathbf{E}_{k}$. If $f$ is real analytic, $f_{\mathbf{C}}: \mathbf{C}^{k} \rightarrow \mathbf{C}$ denotes the complexification of $f$.

Received by the editors January 5, 2000.

1991 Mathematics Subject Classification. Primary 58K40; Secondary 32S05.

Key words and phrases. Non-isolated singularities, line singularities, infinite determinacy, Lojasiewicz inequality. 
The following results are classical (a good reference is [Wa]).

Theorem 1. Suppose $f \in \mathbf{E}_{n}$. The following are equivalent:

(1) $f$ is finitely $\mathcal{R}$-determined;

(2) $J(f) \supset \mathbf{m}_{n}^{l}$ for some $l \in \mathbf{N}$;

(3a) (if $f$ is analytic) $f_{\mathbf{C}}$ has an isolated singularity at 0 .

Theorem 2. Suppose $f \in \mathbf{E}_{n}$. The following are equivalent:

(1) $f$ is infinitely $\mathcal{R}$-determined;

(2) $J(f) \supset \mathbf{m}_{n}^{\infty}$;

(3a) (if $f$ is analytic) $f$ has an isolated singularity at 0 .

The assumption in (3a) of Theorem 2 that $f$ be analytic can be dropped if we replace the condition of isolated singularity by a Łojasiewicz inequality. One says that a continuous germ $g$ at 0 is Eojasiewicz on $A$ at $S$ if for one (and hence for every) representative $G$ of $g$, there is a neighborhood $U$ of 0 and constants $C, r>0$ such that $|G(x)| \geq C d(x, S)^{r}$ for all $x \in U \cap A$, where $d(x, S)=\inf _{y \in S}|x-y|$ (necessarily $S$ contains $g^{-1}(0)$ ). We omit saying "on $A$ " if $A$ contains a neighborhood of 0 . A finitely generated ideal $I$ is Łojasiewicz at $S$ if it contains an element which is Eojasiewicz at $S$. As pointed out in section V.4 of [To], it is equivalent to check for any set of generators of the ideal whether the sum of the squares of the generators or the sum of the absolute values of the generators is Lojasiewicz at $S$. Then Theorem 2 is true if we replace condition (3a) by

$$
J(f) \text { is Lojasiewicz at } 0 .
$$

It is a well-known result of Łojasiewicz that every analytic germ at 0 is Łojasiewicz at its zero set. Hence condition (3) reduces to condition (3a) when $f$ is analytic.

Before stating the analogous theorems for isolated line singularities, we need some more notation and some preliminary results.

Let $z=(x, y)=\left(x, y_{1}, \ldots, y_{n}\right)=\left(z_{0}, z_{1}, \ldots, z_{n}\right)$ be the coordinate system of $\mathbf{R}^{n+1}$, where $x \in \mathbf{R}$ and $y \in \mathbf{R}^{n}$, and let $|y|=\sum_{i=1}^{n}\left|y_{i}\right|,|z|=\sum_{i=0}^{n}\left|z_{i}\right|$. Let $f:\left(\mathbf{R}^{n+1}, 0\right) \rightarrow(\mathbf{R}, 0)$ be a germ of a smooth function with a smooth 1-dimensional critical set $\Sigma(f)$. Necessarily $f=0$ on $\Sigma(f)$. After a change of coordinates we can assume $\Sigma(f)=L=\{(x, y): y=0\}$, so $f(z) \in(y)^{2} \mathbf{E}_{n+1}$, where $(y)^{2} \mathbf{E}_{n+1}$ denotes the ideal in $\mathbf{E}_{n+1}$ generated by all $y_{i} y_{j}, 1 \leq i, j \leq n$. For any ring $R$, let $\mathbf{M}(R, n)$ denote the space of all $n \times n$ matrices with entries in $R$, and let $\mathbf{S}(R, n)$ denote the subspace of $\mathbf{M}(R, n)$ consisting of all symmetric matrices.

Let $\mathcal{R}_{L}$ denote the subgroup of all local diffeomorphisms $\phi$ at 0 on $\mathbf{R}^{n+1}$ which fix the origin and leave the $x$-axis invariant, i.e., $\phi(0)=0$ and $\phi(L)=L$. The group $\mathcal{R}_{L}$ acts on $(y)^{2} \mathbf{E}_{n+1}$ by composing on the right. Let $\mathcal{R}_{L} \cdot f$ denote the orbit of $f \in(y)^{2} \mathbf{E}_{n+1}$.

Definition. $f$ and $g$ in $(y)^{2} \mathbf{E}_{n+1}$ are said to be $\mathcal{R}_{L}$-equivalent if there is some $\phi \in \mathcal{R}_{L}$ such that $f=g \circ \phi$, i.e., $\mathcal{R}_{L} \cdot f=\mathcal{R}_{L} \cdot g$.

Definition. $f \in(y)^{2} \mathbf{E}_{n+1}$ is $k$-determined in $(y)^{2} \mathbf{E}_{n+1}$ if $f+(y)^{2} \mathbf{m}_{n+1}^{k-1} \subset \mathcal{R}_{L} \cdot f$, $f$ is finitely determined in $(y)^{2} \mathbf{E}_{n+1}$ if $f$ is $k$-determined in $(y)^{2} \mathbf{E}_{n+1}$ for some $k$, and $f$ is $\infty$-determined in $(y)^{2} \mathbf{E}_{n+1}$ if $f+(y)^{2} \mathbf{m}_{n+1}^{\infty} \subset \mathcal{R}_{L} \cdot f$. 
It is shown in Si] that

$$
\tau(f)=\left\{a \frac{\partial f}{\partial x}+\sum_{l} b_{l} \frac{\partial f}{\partial y_{l}}: a \in \mathbf{m}_{n+1}, b_{l} \in(y) \mathbf{E}_{n+1}, 1 \leq l \leq n\right\}
$$

is the tangent space at $f$ to the orbit $\mathcal{R}_{L} \cdot f$.

Let $f=\sum_{i, j} y_{i} y_{j} f_{i j}$, with $f_{i j}=f_{j i}$. Let $D(x, y)=\operatorname{det}\left(f_{i j}\right)$ and, by abuse of notation, let $D(x)=D(x, 0) ; D(x)$ is the determinant of the Hessian matrix of $f$ with respect to $y$ on $L$, i.e., the matrix of second partials of $f$ with respect to the $y$ variables, evaluated on $L$. Thus $D(x)$ is independent of the choice of the $f_{i j}$ 's.

Definition. $f \in \mathbf{E}_{n+1}$ has a real isolated line singularity if:

(1) $\Sigma(f)$ is a nonsingular curve through 0 ;

(2) $J(f)$ is Eojasiewicz at $\Sigma(f)$; and

(3) $D \mid \Sigma(f)$ is Lojasiewicz at 0 .

Remark. The second condition in the above definition does not imply the third by the following example. Obviously the third one can't imply the second.

Examples. Let $f(x, y)=x\left(y_{1}^{4}+y_{2}^{4}\right)$ and let $g(x, y)=y_{1}^{3}+y_{2}^{2}$. Both have singular set the $x$-axis, and their Jacobian ideals are Eojasiewicz at the $x$-axis since they are analytic. In both cases, $D(x)=0$ for all $x$, so condition (3) of the definition fails.

Here are some functions which are isolated line singularities: $y^{2}, x y^{2}, x^{2} y^{2}$, $x^{2} y^{2}+y^{r}, r \geq 3$. The following function is a real isolated line singularity, but its complexification is not an isolated line singularity: $\left(y_{1}^{2}+y_{2}^{2}\right)\left(x^{2}+y_{1}^{2}+y_{2}^{2}\right)$.

Lemma 1.1. Assuming that $\Sigma(f)=\{y=0\}$, condition (2) in the definition of real isolated line singularity can be replaced by: for every $r>0, J(f)$ is Eojasiewicz at 0 on $\left\{z=(x, y):|y| \geq|x|^{r}\right\}$.

Proof. It is routine to see that (2) implies the condition of this Lemma. We shall show that (3) implies:

(*) there is some $r>0$ such that $J(f)$ is Łojasiewicz at $L$ on $H_{r}=\left\{|y| \leq|x|^{r}\right\}$.

Hence (3) and the condition of this Lemma together imply (2).

Suppose that (3) holds and that $(*)$ fails. Then, for each $p \in \mathbf{N}, J(f)$ fails to be Eojasiewicz at $L$ on $H_{p}$. Let $d_{y} f=\left(\frac{\partial f}{\partial y_{1}}, \ldots, \frac{\partial f}{\partial y_{n}}\right)$ and let $d_{y}^{2} f$ denote the Hessian matrix of $f$ with respect to $y$. Then there exists a sequence $z_{p}=\left(x_{p}, y_{p}\right) \rightarrow 0$ with $\left|y_{p}\right| \leq\left|x_{p}\right|^{p}$ such that $\left|d_{y} f\left(z_{p}\right)\right| \leq\left|y_{p}\right|^{p}$. Let $S_{p}$ denote the line segment joining $w_{p}=\left(x_{p}, 0\right)$ to $z_{p}$, let $r$ be the distance from $L$, and let $\frac{\partial g}{\partial r}$ denote the derivative of any function $g$ with respect to $r$. Since $d_{y} f\left(w_{p}\right)=0$, the Mean Value Theorem applied to $\frac{\partial f}{\partial y_{i}} \mid S_{p}$ implies that there exist $z_{p, i}=\left(x_{p}, y_{p, i}\right) \in S_{p}$ such that

$$
\begin{aligned}
\left|\frac{\partial^{2} f}{\partial r \partial y_{i}}\left(z_{p, i}\right)\right| & =\left|\frac{\partial f}{\partial y_{i}}\left(z_{p}\right)\right| /\left|y_{p}\right| \\
& \leq\left|y_{p}\right|^{p-1} \leq\left|x_{p}\right|^{p(p-1)}
\end{aligned}
$$

Note that $\frac{\partial}{\partial r} d_{y} f=u_{r} \cdot d_{y}^{2} f$, where $u_{r}$ is the unit radial vector in $L^{\perp}$. It is not hard to see that $D(x)=\operatorname{det} d_{y}^{2} f(x, 0)$ Łojasiewicz at 0 implies that $u_{r} \cdot d_{y}^{2} f(x, 0)$ is Eojasiewicz at 0 ; hence, there is an $R>0$ such that $\left|\frac{\partial}{\partial r} d_{y} f\left(w_{p}\right)\right| \geq\left|x_{p}\right|^{R} \geq$ 
$2 n\left|x_{p}\right|^{p(p-1)}$ for $p$ sufficiently large. There exist $z_{p, i}^{\prime} \in S_{p}$ such that

$$
\sum_{i}\left|\frac{\partial^{3} f}{\partial r^{2} \partial y_{i}}\left(z_{p, i}^{\prime}\right)\right|=\sum_{i} \frac{\left|\frac{\partial^{2} f}{\partial r \partial y_{i}}\left(z_{p, i}\right)-\frac{\partial^{2} f}{\partial r \partial y_{i}}\left(w_{p}\right)\right|}{\left|y_{p, i}\right|} \geq \frac{\left|x_{p}\right|^{R}}{2\left|x_{p}\right|^{p}} \rightarrow \infty
$$

as $p \rightarrow \infty$. This is impossible since $f$ is $C^{3}$.

Theorem 3 ( $\underline{\text { Si] }]) . ~ F o r ~} f \in(y)^{2} \mathbf{E}_{n+1}$, the following conditions are equivalent:

(1) $f$ is finitely determined in $(y)^{2} \mathbf{E}_{n+1}$.

(2) $\tau(f) \supset(y)^{2} \mathbf{m}_{n+1}^{k}$ for some $k$.

(3) $J(f) \supset(y) \mathbf{m}_{n+1}^{k}$ for some $k$.

(4a) ( $f$ analytic) $f_{\mathbf{C}}$ has an isolated line singularity.

Actually Siersma only considered complex analytic functions, but it is easy to check that (1), (2) and (3) are still equivalent in the smooth case.

Siersma's result has also been generalized to the case in which $\Sigma(f)$ is a curve with a singularity at 0 (see $\mathrm{Pe}$ and [zMats]).

The principal result of this paper extends Theorem 3 to real isolated line singularities and infinite determinacy:

Theorem 4. For $f \in(y)^{2} \mathbf{E}_{n+1}$, the following conditions are equivalent:

(1) $f$ is infinitely determined in $(y)^{2} \mathbf{E}_{n+1}$.

(2) $\tau(f) \supset(y)^{2} \mathbf{m}_{n+1}^{\infty}$.

(3) $J(f) \supset(y) \mathbf{m}_{n+1}^{\infty}$.

(4) $f$ has a real isolated line singularity.

$(4) \Rightarrow(3)$ is proved in $\S 2,(3) \Rightarrow(2)$ is trivial, $(2) \Rightarrow(1)$ is proved in $\S 3$ and $(1) \Rightarrow(4)$ is proved in $\S 4$.

\section{$\S 2$. The Jacobian of $f$}

Let $\mathcal{M}$ be a finitely generated $\mathbf{E}_{k}$ submodule of $\mathbf{E}_{k}^{p}$. A set of generators $f_{1}, \ldots, f_{r}$ of $\mathcal{M}$ gives rise to a $p \times r$ matrix $M$ whose columns are the $f_{j}$ 's; then $M\left(\mathbf{E}_{k}^{r}\right)=\mathcal{M}$. Let $F_{0}(M)$ denote the ideal in $\mathbf{E}_{k}$ generated by the $p \times p$ minors of $M$. It is easy to see that this ideal is independent of the choice of generators (use the Cauchy-Binet formula for the determinant of the product of two matrices) - it is called the 0th Fitting ideal of the module $\mathcal{M}$.

Lemma 2.1. $\mathcal{M} \supset\left(\mathbf{m}_{k}^{\infty}\right)^{p}$ iff $F_{0}(\mathcal{M}) \supset \mathbf{m}_{k}^{\infty}$.

Proof. "if": Let $M$ be a matrix whose columns generate $\mathcal{M}$. For any $p \times p$ matrix $A, A(\operatorname{adj} A)=(\operatorname{det} A) I$; hence $A\left(\mathbf{E}_{k}^{p}\right)=\operatorname{det} A \cdot \mathbf{E}_{k}^{p}$. Applying this to the $p \times p$ submatrices of $M$, we conclude $\mathcal{M} \supset F_{0}(\mathcal{M}) \mathbf{E}_{k}^{p}$.

"only if": Pick $u \in \mathbf{m}_{k}^{\infty}$. There exist $u_{1}, \ldots, u_{p} \in \mathbf{m}_{k}^{\infty}$ such that $u=u_{1} \cdots \cdots u_{p}$ (see for example [T] $]$ ). Let $A$ be the diagonal matrix with $u_{1}, \ldots, u_{p}$ on the diagonal. By assumption, there is an $r \times p$ matrix $N$ such that $A=M N$. By the Cauchy-Binet formula, $u=\operatorname{det} A$ is in $F_{0}(\mathcal{M})$.

Let $\psi: \mathbf{E}_{n+1}^{n} \rightarrow(y) \mathbf{E}_{n+1}$ be the map defined by $\left(f_{1}, f_{2}, \cdots, f_{n}\right) \mapsto \sum_{i=1}^{n} y_{i} f_{i}$. Then ker $\psi$ is the module of relations among the functions $y_{1}, \ldots, y_{n} \in \mathbf{E}_{n+1}$. Let $e_{i} \in \mathbf{E}_{n+1}^{n}$ have a 1 in the $i$-th component and 0's elsewhere, for $1 \leq i \leq n$.

Lemma 2.2. $\operatorname{ker} \psi$ is generated by the trivial relations $\left\{y_{i} e_{j}-y_{j} e_{i}: 1 \leq j<i \leq n\right\}$. 
Proof. Let $\mathbf{O}_{k}$ denote the ring of analytic germs at 0 in $\mathbf{R}^{k}$ and let $\alpha: \mathbf{O}_{n+1}^{n} \rightarrow$ $(y) \mathbf{O}_{n+1}$ be the map defined by $\left(f_{1}, f_{2}, \cdots, f_{n}\right) \mapsto \sum_{i=1}^{n} y_{i} f_{i}$.

Because $y_{1}, \ldots, y_{n}$ is a regular sequence in $\mathbf{O}_{n+1}$, the analytic relations ker $\alpha$ are generated by the trivial relations (see I.5.1 of [To]). But $\mathbf{E}_{n+1}$ is a flat $\mathbf{O}_{n+1^{-}}$ module (Corollary VI.1.3 of [To]). It follows from Proposition I.4.2 of [To that $(\operatorname{ker} \alpha) \otimes \mathbf{O}_{n+1} \mathbf{E}_{n+1}=\operatorname{ker}\left(\alpha \otimes_{\mathbf{O}_{n+1}} \mathbf{E}_{n+1}\right)=\operatorname{ker} \psi$, i.e., the smooth relations $\operatorname{ker} \psi$ are also generated by the trivial relations.

Let $R(f)$ denote the matrix whose columns are these generators of ker $\psi$.

Next we wish to determine $\psi^{-1} J(f)$ for $f \in(y)^{2} \mathbf{E}_{n+1}$. We write $f=\sum_{i=1}^{n} y_{i} f_{i}$, and $f_{i}=\sum_{j=1}^{n} y_{j} f_{i j}$ with $f_{i j}=f_{j i}$. Then $\frac{\partial f}{\partial x}=\sum_{i=1}^{n} y_{i} \frac{\partial f_{i}}{\partial x}$ and $\frac{\partial f}{\partial y_{j}}=f_{j}+$ $\sum_{i=1}^{n} y_{i} \frac{\partial f_{i}}{\partial y_{j}}=\sum_{i=1}^{n} y_{i}\left(\frac{\partial f_{i}}{\partial y_{j}}+f_{i j}\right)$. Let $\mathcal{M}(f)$ be the module generated by the columns

$$
\left(\begin{array}{c}
\frac{\partial f_{1}}{\partial x} \\
\frac{\partial f_{2}}{\partial x} \\
\cdot \\
\cdot \\
\cdot \\
\frac{\partial f_{n}}{\partial x}
\end{array}\right),\left(\begin{array}{c}
\frac{\partial f_{1}}{\partial y_{1}}+f_{11} \\
\frac{\partial f_{2}}{\partial y_{1}}+f_{21} \\
\cdot \\
\cdot \\
\cdot \\
\frac{\partial f_{n}}{\partial y_{1}}+f_{n 1}
\end{array}\right), \quad \cdots,\left(\begin{array}{c}
\frac{\partial f_{1}}{\partial y_{n}}+f_{1 n} \\
\frac{\partial f_{2}}{\partial y_{n}}+f_{2 n} \\
\cdot \\
\cdot \\
\cdot \\
\frac{\partial f_{n}}{\partial y_{n}}+f_{n n}
\end{array}\right),
$$

and let $M(f)$ be the corresponding matrix. Let $M(f) \mid R(f)$ be the matrix formed by adjoining these two matrices (so this matrix generates the submodule $\mathcal{M}(f)+\operatorname{ker} \psi$ ).

Thus

$$
\left(y_{1}, \ldots, y_{n}\right) \cdot M(f)=\left(\frac{\partial f}{\partial x}, \frac{\partial f}{\partial y_{1}}, \ldots, \frac{\partial f}{\partial y_{n}}\right)
$$

generates $J(f)$. Therefore

$$
\psi^{-1} J(f)=\mathcal{M}(f)+\operatorname{ker} \psi .
$$

Let $I(f)=F_{0}(\mathcal{M}(f)+\operatorname{ker} \psi)$.

Proposition 2.3. $J(f) \supset(y) \mathbf{m}_{n+1}^{\infty}$ if, and only if, $I(f)$ is Lojasiewicz at 0 .

Proof. $J(f) \supset(y) \mathbf{m}_{n+1}^{\infty}$ iff $\mathcal{M}(f)+\operatorname{ker} \psi \supset \psi^{-1}\left((y) \mathbf{m}_{n+1}^{\infty}\right)=\mathbf{m}_{n+1}^{\infty}\left(\mathbf{E}_{n+1}^{n}\right)+\operatorname{ker} \psi$ iff $\mathcal{M}(f)+\operatorname{ker} \psi \supset \mathbf{m}_{n+1}^{\infty}\left(\mathbf{E}_{n+1}^{n}\right)$ iff $I(f) \supset \mathbf{m}_{n+1}^{\infty}$ (by Lemma 2.1) iff $I(f)$ is Łojasiewicz at 0 (by Proposition V.4.3 of [To]).

Proof of $(4) \Rightarrow(3)$. By assumption, $\left|\frac{\partial f}{\partial x}\right|+\sum\left|\frac{\partial f}{\partial y_{j}}\right|$ is Eojasiewicz at $L$, and $D(x)=$ $D(x, 0)=\operatorname{det}\left(f_{i, j}(x, 0)\right)$ is Lojasiewicz at 0 . By Proposition 2.3, it suffices to prove that $I(f)$ is Lojasiewicz at 0 .

For each $i$ and $j, 1 \leq i \leq n$ and $1 \leq j \leq n+1$, let $M_{i, j}$ denote the $n \times n$ submatrix of $M(f) \mid R(f)$ whose first column is the $j$-th column of $M(f)$ and whose other columns are the relations $y_{i} e_{k}-y_{k} e_{i}, 1 \leq k<i$, and $y_{k} e_{i}-y_{i} e_{k}, i<k \leq n$. Then $\operatorname{det} M_{i, 1}= \pm y_{i}^{n-2} \frac{\partial f}{\partial x}$ and $\operatorname{det} M_{i, j+1}= \pm y_{i}^{n-2} \frac{\partial f}{\partial y_{j}}$ for $1 \leq j \leq n$. Thus 
$\Delta=\sum\left|M_{i, j}\right|=\left(\sum\left|y_{i}\right|^{n-2}\right)\left(\left|\frac{\partial f}{\partial x}\right|+\sum\left|\frac{\partial f}{\partial y_{j}}\right|\right)$ is a sum of absolute values of elements of $I(f)$ which is Łojasiewicz at $L$. It follows that $\Delta$ is Łojasiewicz at 0 on the set $\left\{(x, y):|y| \geq C|x|^{r}\right\}$, for any positive constants $C$ and $r$. We need to find an element of $I(f)$ which is Lojasiewicz on the complementary set

$$
H_{r, C}=\left\{(x, y):|y| \leq C|x|^{r}\right\} .
$$

Let $A=\left(\frac{\partial f_{i}}{\partial y_{j}}+f_{i j}\right)$. Since $\frac{\partial f_{i}}{\partial y_{j}}=f_{i j}+\sum_{k} y_{k} \frac{\partial f_{i k}}{\partial y_{j}}, A=\left(2 f_{i j}+\sum_{k} y_{k} \frac{\partial f_{i k}}{\partial y_{j}}\right)$; hence $\operatorname{det} A=2^{n}(D(x, y)+b(x, y))$ for some $b \in(y) \mathbf{E}_{n+1}$. Let $b=\sum_{i=1}^{n} y_{i} b_{i}$.

Also $D(x, y)-D(x) \in(y) \mathbf{E}_{n+1}$, so it can be written as $\sum_{i=1}^{n} y_{i} a_{i}$. Thus

$$
D(x)=2^{-n} \operatorname{det} A-\sum_{i=1}^{n} y_{i}\left(a_{i}+b_{i}\right) .
$$

By assumption, there exist $C, r>0$ such that

$$
C|x|^{r} \leq|D(x)| \leq 2^{-n}|\operatorname{det} A|+\sum\left|y_{i}\right|\left|a_{i}+b_{i}\right|
$$

near 0 . There is a constant $L>0$ such that $\left|a_{i}+b_{i}\right| \leq L$ near 0 . Consequently, on the set $H_{r, C /(2 L)}$,

$$
\sum\left|y_{i}\right|\left|a_{i}+b_{i}\right| \leq L \sum\left|y_{i}\right| \leq \frac{C}{2}|x|^{r},
$$

which implies that $2^{-n}|\operatorname{det} A| \geq \frac{C}{2}|x|^{r}$.

We conclude that $\Delta+|\operatorname{det} A|$ is a sum of absolute values of elements of $I(f)$ which is Łojasiewicz at 0 , which implies that $I(f)$ is Łojasiewicz at 0 , as desired.

§3. The tangent space $\tau(f)$ to the orbit of $f$ in $(y)^{2} \mathbf{E}_{n+1}$

Suppose that $f, g \in(y)^{2} \mathbf{E}_{n+1}$ and $j^{\infty} f(0)=j^{\infty} g(0)$. Let $u=g-f$. It follows from Proposition V.2.3 of [To] that there exist $u_{i j} \in \mathbf{m}_{n+1}^{\infty}$ such that $u=\sum_{i, j} y_{i} y_{j} u_{i j}$ with $u_{i j}=u_{j i}$. Let $F=f+t u, 0 \leq t \leq 1$. For any $t_{0} \in[0,1]$, let

$$
\tau^{*}(F)_{\left(0, t_{0}\right)}=\left\{a \frac{\partial F}{\partial x}+\sum_{l=1}^{n} b_{l} \frac{\partial F}{\partial y_{l}}: a \in \mathbf{m}_{n+1} \mathbf{E}_{n+2}\left(0, t_{0}\right), b_{l} \in(y) \mathbf{E}_{n+2}\left(0, t_{0}\right)\right\},
$$

where $\mathbf{E}_{n+2}\left(0, t_{0}\right)$ denotes the smooth germs at $\left(0, t_{0}\right) \in \mathbf{R}^{n+2}$.

We are trying to show that $g$ is $\mathcal{R}_{L}$-equivalent to $f$. It suffices to show that there exists for each $t \in[0,1]$ an $h_{t} \in \mathcal{R}_{L}$ such that $F \circ\left(h_{t}, t\right)=f$. A standard argument (see [Math] or [Mart]) shows that we can find $h_{t}$ if, for each $t_{0} \in[0,1], u=\partial F / \partial t \in$ $\tau^{*}(F)_{\left(0, t_{0}\right)}$. Thus, it suffices to show that $\tau^{*}(F)_{\left(0, t_{0}\right)} \supset(y)^{2} \mathbf{m}_{n+1}^{\infty} \mathbf{E}_{n+2}\left(0, t_{0}\right)$.

By assumption, $\tau(f) \supset(y)^{2} \mathbf{m}_{n+1}^{\infty}$. Therefore,

$$
\tau(f) \mathbf{E}_{n+2}\left(0, t_{0}\right) \supset(y)^{2} \mathbf{m}_{n+1}^{\infty} \mathbf{E}_{n+2}\left(0, t_{0}\right) .
$$

If $\eta \in \tau^{*}(F)_{\left(0, t_{0}\right)}$, then there exist $a \in \mathbf{m}_{n+1} \mathbf{E}_{n+2}\left(0, t_{0}\right)$ and $b_{l} \in(y) \mathbf{E}_{n+2}\left(0, t_{0}\right)$, $1 \leq l \leq n$, such that

$$
\eta=a \frac{\partial F}{\partial x}+\sum_{l} b_{l} \frac{\partial F}{\partial y_{l}}
$$


Let

$$
\eta_{1}=a \frac{\partial f}{\partial x}+\sum_{l} b_{l} \frac{\partial f}{\partial y_{l}} \in \tau(f) \mathbf{E}_{n+2}\left(0, t_{0}\right)
$$

Then

$$
\eta-\eta_{1}=a t \frac{\partial u}{\partial x}+\sum_{l} b_{l} t \frac{\partial u}{\partial y_{l}} \in(y)^{2} \mathbf{m}_{n+1}^{\infty} \mathbf{E}_{n+2}\left(0, t_{0}\right),
$$

as required.

\section{$\S 4$. The Proof OF $(1) \Rightarrow(4)$}

In this section we assume that $f$ is infinitely determined in $(y)^{2} \mathbf{E}_{n+1}$.

Let $\phi=\left(\phi_{0}, \phi_{1}, \ldots, \phi_{n}\right) \in \mathcal{R}_{L}$; then there exist $a, a_{i j}, b_{j} \in \mathbf{E}_{n+1}$ such that $\phi_{i}=$ $\sum_{j=1}^{n} a_{i j} y_{j}, 1 \leq i \leq n$, and $\phi_{0}=a x+\sum_{j=1}^{n} y_{j} b_{j}$. Write $\psi=\left(\phi_{1}, \ldots, \phi_{n}\right)^{T}$ (where $T$ denotes "transpose"), $\beta=\left(b_{1}, \ldots, b_{n}\right)$ and $A=\left(a_{i j}\right)$. Then $\phi=\left(\begin{array}{c}\phi_{0} \\ \psi\end{array}\right)=\left(\begin{array}{cc}a & \beta \\ 0 & A\end{array}\right)\left(\begin{array}{l}x \\ y\end{array}\right)$. Since $\phi$ is a diffeomorphism, the matrix $\left(\begin{array}{ll}a & \beta \\ 0 & A\end{array}\right)$ is invertible, and its inverse is

$$
\left(\begin{array}{cc}
a^{-1} & -a^{-1} \beta A^{-1} \\
0 & A^{-1}
\end{array}\right)
$$

Lemma 4.1. Let $\phi, A$ and $a_{i j}$ be as above. If $f=\sum_{i, j} f_{i j} y_{i} y_{j}, g=f \circ \phi$ and $\left(g_{i j}\right)=A^{T}\left(f_{i j} \circ \phi\right) A$, then $g=\sum_{i, j} g_{i j} y_{i} y_{j}$.

Proof.

$$
\begin{aligned}
g & =\sum_{i, j} \phi_{i} \phi_{j}\left(f_{i j} \circ \phi\right) \\
& =\sum_{i j}\left(\sum_{k} a_{i, k} y_{k}\right)\left(\sum_{l} a_{j l} y_{l}\right)\left(f_{i j} \circ \phi\right) \\
& =\sum_{k, l}\left(\sum_{i, j} a_{i k}\left(f_{i j} \circ \phi\right) a_{j l}\right) y_{k} y_{l} \\
& =\sum_{k, l} g_{k l} y_{k} y_{l} .
\end{aligned}
$$

Corollary. If $f$ and $g$ are $\mathcal{R}_{L}$-equivalent, then

$$
\operatorname{det}\left(g_{i j}(x, 0)\right) \in \mathbf{m}_{1}^{\infty} \quad \text { if, and only if, } \quad D(x) \in \mathbf{m}_{1}^{\infty} .
$$

Lemma 4.2. If the sequence $\left\{x_{p}\right\}$ satisfies (1) $0<x_{p}<\frac{1}{2}$ and (2) $x_{p+1}<\frac{1}{3} x_{p}$, and the sequence $\left\{A_{p}=\left(a_{i j}^{p}\right)\right\}$ of symmetric matrices satisfies $\left|a_{i j}^{p}\right|<x_{p}^{p}$ for all $i, j$, then there is some $u \in(y)^{2} \mathbf{m}_{n+1}^{\infty}$ such that $\frac{\partial^{2} u}{\partial y_{i} \partial y_{j}}\left(x_{p}, 0\right)=a_{i j}^{p}$ for all $i, j$.

Proof. Let $B_{p}$ be the interval $\left(\frac{2}{3} x_{p}, \frac{4}{3} x_{p}\right)$; then $B_{p} \cap B_{q}=\emptyset$ for $p \neq q$. Let $\mu_{p}$ be a smooth function on $\mathbf{R}$ with the following properties: (1) $\mu_{p}\left(x_{p}\right)=1, \mu_{p}=0$ outside $B_{p}$ and (2) there exist positive constants $C_{k}$ depending only on $k$ (not on $x_{p}$ ) such that $\left|\mu_{p}^{(k)}(x)\right| \leq \frac{C_{k}}{x_{p}^{k}}$ for every $k \in \mathbf{N}$ (see Lemma IV.3.3 of [To]). 
Now let $u(z)=\sum_{p=1}^{\infty} \sum_{i, j=1}^{n} y_{i} y_{j} \mu_{p}(x) \frac{a_{i j}^{p}}{2}$, for $z=(x, y)$. Then $u$ is smooth outside the hyperplane $x=0$. Let $k$ denote the multi-index $\left(k_{0}, k^{\prime}\right)$, with $k^{\prime}=$ $\left(k_{1}, \ldots, k_{n}\right)$. Let $e_{i}$ denote the $n$-tuple with 1 in the $i$-th place, 0 elsewhere. Then

$$
\frac{\partial^{|k|} u}{\partial z^{k}}(z)=\left\{\begin{array}{lll}
0, & x \notin \cup_{p} B_{p} \quad \text { or } \quad\left|k^{\prime}\right|>2, \\
\sum_{p} \sum_{i, j} y_{i} y_{j} \mu_{p}^{\left(k_{0}\right)}(x) \frac{a_{i j}^{p}}{2}, & x \in B_{p} \quad \text { and } \quad k^{\prime}=0 \\
\sum_{p} \sum_{j=1}^{n} y_{j} \mu_{p}^{\left(k_{0}\right)}(x) a_{i j}^{p}, & x \in B_{p} \quad \text { and } \quad k^{\prime}=e_{i}, \\
\sum_{p} \sum_{j=1}^{n} \mu_{p}^{\left(k_{0}\right)}(x) a_{i j}^{p}, & x \in B_{p} & \text { and } \quad k^{\prime}=e_{i}+e_{j} .
\end{array}\right.
$$

In particular, $\frac{\partial^{2} u}{\partial y_{i} \partial y_{j}}\left(x_{p}, 0\right)=a_{i j}^{p}$ and $u(x, 0)=\frac{\partial u}{\partial y_{i}}(x, 0)=0$ for $x \neq 0$. Since

$$
\left|\mu_{p}^{(k)}(x) a_{i j}^{p}\right| \leq \frac{C_{k}}{x_{p}^{k}} x_{p}^{p}=C_{k} x_{p}^{p-k},
$$

from $(*)$ it follows that $\lim _{z \rightarrow 0} \frac{\partial^{|k|} u}{\partial z^{k}}(z)=0$. By the Lemma on page 87 of [Mart], $u \in C^{\infty}\left(\mathbf{R}^{n+1}\right)$. So $u \in(y)^{2} \mathbf{m}_{n+1}^{\infty}$.

Lemma 4.3. There is a sequence $x_{p} \neq 0, x_{p} \rightarrow 0$, such that $D\left(x_{p}\right) \neq 0$.

Proof. Assume $D(x)=0$ for all $x$. Choose a sequence $x_{p}>0$ as in Lemma 4.2. Let $\Sigma=\{A \in \mathbf{M}(\mathbf{R}, n): \operatorname{det} A=0\}$. Choose $A_{p}=\left(a_{i j}^{p}\right) \in \mathbf{S}(\mathbf{R}, n) \backslash \Sigma$ with $\left|a_{i j}^{p}-f_{i j}\left(x_{p}, 0\right)\right|<\left|x_{p}\right|^{p}$ for all $i, j$. By Lemma 4.2, there exists $u \in(y)^{2} \mathbf{m}_{n+1}^{\infty}$ with $\frac{\partial^{2} u}{\partial y_{i} \partial y_{j}}\left(x_{p}, 0\right)=a_{i j}^{p}-f_{i j}\left(x_{p}, 0\right)$. Then $\operatorname{det}\left((f+u)_{i j}\left(x_{p}, 0\right)\right)=\operatorname{det} A_{p} \neq 0$. By Lemma $4.1, D\left(\phi\left(x_{p}, 0\right)\right) \neq 0$, which is a contradiction.

Note that $D(x)$ is not Łojasiewicz if, and only if, $D(x) \in \mathbf{m}_{1}^{\infty}$.

Lemma 4.4. For each $k$, there is at least one $k \times k$ minor of the matrix $\left(f_{i j}(x, 0)\right)$ which is not flat at 0 . In particular $D(x) \notin \mathbf{m}_{1}^{\infty}$.

Proof. Since $f$ is $\infty$-determined in $(y)^{2} \mathbf{E}_{n+1}, g(z)=f(z)-\sum^{\prime} y_{i} y_{j} f_{i j}(x, 0)$ is $\mathcal{R}_{L^{-}}$ equivalent to $f$, where the summation is taken over the set $\left\{(i, j): f_{i j}(x, 0) \in \mathbf{m}_{1}^{\infty}\right\}$. By the Corollary of Lemma 4.1 we may assume that $f_{i j}(x, 0)$ is either 0 or not in $\mathbf{m}_{1}^{\infty}$. By Lemma 4.3, $D(x)$ is not identically equal to 0 , so at least one of the entries is not 0 .

We will prove the result by induction. The result clearly holds for $k=1$. Assume it holds for $k$. Assume $B=\left(f_{i_{s} j_{t}}(x, 0)\right)$ is a $k \times k$ submatrix of $\left(f_{i j}(x, 0)\right)$, whose determinant is not in $\mathbf{m}_{1}^{\infty}, 1 \leq k<n$. Take any $l \notin\left\{j_{1}, \ldots, j_{k}\right\}$. Look at the matrix

$$
A=\left(\begin{array}{cccc}
f_{1 j_{1}}(x, 0) & \ldots & f_{1 j_{k}}(x, 0) & f_{1 l}(x, 0) \\
\ldots & \ldots & \ldots & \ldots \\
f_{n j_{1}}(x, 0) & \ldots & f_{n j_{k}}(x, 0) & f_{n l}(x, 0)
\end{array}\right) .
$$

For any $m \notin\left\{i_{1}, \ldots, i_{k}\right\}$, let

$$
A_{m}=\left(\begin{array}{cccc}
f_{i_{1} j_{1}}(x, 0) & \ldots & f_{i_{1} j_{k}}(x, 0) & f_{i_{1} l}(x, 0) \\
\ldots & \ldots & \ldots & \ldots \\
f_{i_{k} j_{1}}(x, 0) & \ldots & f_{i_{k} j_{k}}(x, 0) & f_{i_{k} l}(x, 0) \\
f_{m j_{1}}(x, 0) & \ldots & f_{m j_{k}}(x, 0) & f_{m l}(x, 0)
\end{array}\right) .
$$


If $\operatorname{det} A_{m} \in \mathbf{m}_{1}^{\infty}$ for all $m \notin\left\{i_{1}, \ldots, i_{k}\right\}$, then $g=f-\sum_{m \notin\left\{i_{1}, \ldots, i_{k}\right\}} y_{m} y_{l} \frac{\operatorname{det} A_{m}}{\operatorname{det} B}$ is $\mathcal{R}_{L^{-}}$-equivalent to $f$, since $\frac{\operatorname{det} A_{m}}{\operatorname{det} B} \in \mathbf{m}_{1}^{\infty} \subset \mathbf{m}_{n+1}^{\infty}$. Denote the corresponding submatrices of $\left(g_{i j}(x, 0)\right)$ by $A_{m}^{\prime}$ and $A^{\prime}$; then $\operatorname{det} A_{m}^{\prime}=\operatorname{det} A_{m}-\frac{\operatorname{det} A_{m}}{\operatorname{det} B} \operatorname{det} B=0$.

Since $\operatorname{det} B(x) \notin \mathbf{m}_{1}^{\infty}$, $\operatorname{det} B(x) \neq 0$ for small $x \neq 0$. So for these $x$ 's, any row of $A^{\prime}$ is a linear combination of the first $k$ rows of $A_{m}$; hence any maximal minor of $A^{\prime}(x)$ is 0 for all small $x \neq 0$. By continuity it is also 0 at 0 . Therefore $\operatorname{det}\left(g_{i j}(x, 0)\right)=0$ for all small $x$. By Lemma 4.1 this will imply that $D(x)=0$, which contradicts Lemma 4.3.

Proof of $(1) \Rightarrow(4)$. By Lemma 4.4, $D(x)$ must be Eojasiewicz at 0 .

By Lemma 1.1, it suffices to show, for each $r>0$, that $J(f)$ is Eojasiewicz at 0 on $A_{r}=\left\{z:|y| \geq|x|^{r}\right\}$.

Suppose this fails. Then there exists $r>0$ and a sequence $z_{p} \in A_{r}$ such that $\left|d f\left(z_{p}\right)\right| \leq\left|z_{p}\right|^{p}$. Without loss of generality, we can assume $\left|y_{p+1}\right|<\left|y_{p}\right| / 3$ for all $p$. Let $B_{p}$ be the open ball centered at $z_{p}$ of radius $\left|y_{p}\right| / 3$. The $B_{p}$ 's are disjoint, and 0 is the only limit point in the $x$-axis of the union of these balls. By Lemma IV.3.3 of [To, there exists for each $p$ a smooth function $\mu_{p}$ which is 1 on a neighbourhood of $z_{p}$, zero outside $B_{p}$, and satisfying: for each multi-index $I$ there exists a constant $C_{I}$ independent of $z_{p}$ such that $\left|D^{I} \mu_{p}(x)\right| \leq C_{I} /\left|y_{p}\right|^{|I|}$.

By Sard's Theorem there exist regular values $w_{p}$ of $f$ such that $\left|w_{p}-f\left(z_{p}\right)\right| \leq$ $\left|z_{p}\right|^{p}$. Let $g_{p}$ be the linear function such that $g_{p}\left(z_{p}\right)=w_{p}-f\left(z_{p}\right)$ and $d g_{p}\left(z_{p}\right)=$ $-d f\left(z_{p}\right)$. Let $u=\sum_{p} \mu_{p} g_{p}$. Then $u$ is a smooth function, $u \in \mathbf{m}_{n+1}^{\infty}$ and, since $u$ is identically 0 on a neighborhood of $\{y=0, x \neq 0\}, u \in \mathbf{m}_{L}^{\infty} \subset(y)^{2} \mathbf{E}_{n+1}$. But $f+u$ can't be $\mathcal{R}_{L}$-equivalent to $f$, since $(f+u)\left(z_{p}\right)=w_{p}$ is a critical value of $f+u$ but is not a critical value of $f$.

\section{REFERENCES}

[IzMats] S. Izumiya and S. Matsuoka, Notes on smooth function germs on varieties, Proc. Amer. Math. Soc. 97 (1986), 146-150. MR 87g:58013

[Mart] J. Martinet, Singularities of smooth functions and maps, Cambridge University Press, New York, 1982. MR 83i:58018

[Math] J. N. Mather, Stability of $C^{\infty}$ mappings, III: finitely-determined map germs, Inst. Hautes Études Sci. Publ. Math. 35 (1968), 279-308. MR 43:1215a

[Pe] R. Pellikaan, Finite determinacy of functions with non-isolated singularities, Proc. London Math. Soc. 57 (1988), 357-382. MR 89i:32014

[Si] Dirk Siersma, Isolated line singularities, Proc. Sympos. Pure Math. 40 (1983), 485-496. MR 85d:32017

[To] J. C. Tougeron, Idéaux de fonctions différentiables, Springer-Verlag, New York, 1972. MR 55:13472

[Wa] C. T. C. Wall, Finite determinacy of smooth map-germs, Bull. London Math. Soc. 13 (1981), 481-539. MR 83i:58020

[Wi] L. C. Wilson, Infinitely determined map germs, Canad. J. Math. XXXIII, No.3 (1981), 671-684. MR 82k:58023

Department of Mathematics, University of Hawait, Honolulu, Hawail 96822

Department of Mathematics, University of Hawait, Honolulu, Hawail 96822

E-mail address: les@math.hawaii.edu 